\title{
11 Eigene Inhalte im Web teilen
}

Lernziele

In diesem Kapitel lernen Sie, Dateien, die auf sozialen Plattformen angeboten werden, in Ihren eigenen Publikationen zu verwenden und eigene Dateien - Texte, Bilder, Videos - im Netz zu veröffentlichen. Verwendung von Dateien aus dem und publizieren im Netz ist leicht, wenn man die entsprechenden Dienste kennt, die dies kostenlos ermöglichen.

- Präsentationen veröffentlichen mit Slideshare

- Texte mit Scribd veröffentlichen und gegebenenfalls woanders einbinden

- Bilder verwenden von und ablegen auf Flickr

- Videos von Youtube oder Vimeo verwenden oder hochladen

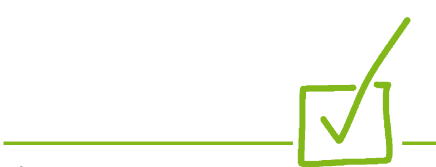

Tipp

- wenn es eher vielfältige Inhalte sind, dann ist ein Weblog das richtige Tool! Die einzelnen Einträge des Blogs dienen der Neuigkeitenbenachrichtigung, größere Texte können - wenn Wordpress verwendet wird - auf statischen Seiten untergebracht werden. Dateien werden hinterlegt und verlinkt. Ein Weblog bietet auch vielfältigere Möglichkeiten, andere Inhalte mit ein paar Mausklicks einzubinden.

- wenn umfangreichere Inhalte zu erwarten sind, die strukturiert und vielfältig miteinander verlinkt werden sollen, ist ein Wiki vorzuziehen.
Zur Sitzung eines Gremiums, als Ergebnis einer Arbeitsgruppe, in einem Workshop oder für einen Vortrag haben Sie Materialien erarbeitet. Seien es Texte, Präsentationen, Bilder oder Videos, Sie möchten sie gern einem weiteren Publikum zugänglich machen. Das sind beispielsweise jene, die an der Sitzung nicht teilnehmen konnten, Stakeholder, die sehen sollen, dass umfangreich und strukturiert gearbeitet wurde, bestimmte Zielgruppen oder gar die weite Öffentlichkeit. Bisher war es so, dass Sie dafür einen Vermittler benötigten, der Ihnen Zugang zu Medien (Zeitung, Zeitschrift, Buch etc.) ermöglichte. Schwieriger Zugang und Zeitverlust waren die Folge. Auf Ihrer Homepage können Sie die Dateien natürlich auch veröffentlichen - nur ist es oft so, dass diese ein Aushängeschild sein soll oder die Richtlinien, was auf die Homepage kann und was nicht, von Ihrer Trägerorganisation eng formuliert sind. Sollten also Restriktionen dieser Art bestehen, können die im Folgenden vorgestellten Dienste eine Alternative darstellen. Mehr noch: Da es Web 2.0-Dienste sind, bringen sie Optionen für die Erschließung und Kommunikation (mit Arbeitskollegen oder mit Zielgruppen) mit sich, die auf einer Homepage zunächst einmal nicht bestehen.

Wir möchten eingangs noch erwähnen, dass wir hier spezialisierte Dienste vorstellen. Vergessen Sie bitte nicht, dass die weniger spezialisierten Web 2.0-Dienste wie Wikis oder Weblogs, die wir ja bereits vorgestellt haben, ebenfalls exzellente Möglichkeiten bieten, Dateien zu veröffentlichen. Bei beiden kann man Dateien ablegen und durch Einträge/Seiten erklärende Texte und Schlagwörter hinzufügen. Wenn Sie also einen Dienst suchen, in dem Ihre Inhalte konsistenter und an einem Ort aufbewahrt sind und der auch weitere Funktionen bezüglich der Wiederauffindbarkeit der Texte bietet, dann sollten Sie an diese Dienste denken!

Die folgenden Dienste bieten Ihnen also die Möglichkeit, Dateien in einem spezifischen Umfeld abzulegen und nutzen zu lassen.

\subsection{Slideshare}

Slideshare ist ein Dienst, der speziell für das Teilen von Präsentationen gegründet wurde. Allerdings sieht man in letzter Zeit immer häufiger, dass Nutzer auch andere Texte hochladen. Sie haben also die Wahl! Entweder veröffentlichen Sie hier die Präsentationen und bei Scibd (s. nächster Abschnitt dieses Kapitels) die Texte oder richten sich auf Slideshare [www.slideshare.net] einen Account ein, in dem Sie alles anbieten. 


\subsubsection{Slideshare als Ressource}

Sie können aber zunächst Slideshare dazu nutzen, um Inhalte bei sich einzubinden, etwa, wenn Sie auf eine gute Präsentation zu einem aktuellen Thema gestoßen sind, das gerade in Ihrer Institution eine Rolle spielt. Beispielsweise haben Sie mit dem Stichwort „Kulturmanagement“ recherchiert und bekommen folgende Ergebnisliste:

\section{\begin{tabular}{l|l}
\hline Slideshare kulturmanagement & Search
\end{tabular}

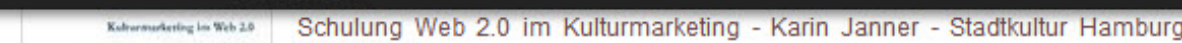

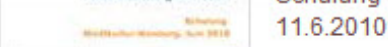 \\ from karinjanner, 1 year ago in Business \& Mgmt \\ 123 slides | 4 favorites}

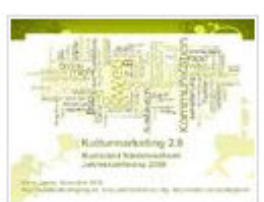

Web 2.0 im Kulturmarketing - Karin Janner Vortrag Musikland Niedersachsen

Vortrag Web $2.0 \mathrm{im}$ Kulturmarketing, karin Janner, auf Jahreskonferen Musikland Niedersachsen, November 2009

from karinjanner, 2 years ago in Business \& Mgmt

66 slides $\mid 3$ favorites

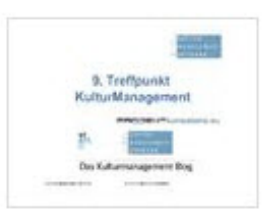

9. Treffpunkt KulturManagement "Arbeitsmarkt Kulturmanagement"

Berufsfelder an der Schnittstelle zwischen Kultur und Management sind vol einem dynamischen Wandel geprägt. Viele Kulturbetriebe setzen dabei au

from KulturmanagementNet, 1 year ago in Business \& Mgmt

33 slides | Download $\Perp$

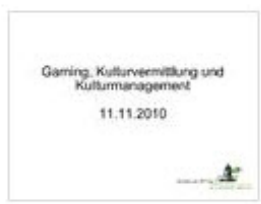

\section{Gaming und Kulturmanagement}

Vortrag zu Gaming und Kulturmanagement am KMM Hamburg

from crocksberlin, 1 year ago in Education

45 slides | Download $\_$ 
Wenn Sie jetzt eine bestimmte Präsentation ausgewählt haben und weitergeben wollen, haben Sie die Option, auf Ihrer Homepage oder in Ihrem Weblog mit einem Link darauf hinzuweisen. Nun wissen Sie aber, dass nur ein Bruchteil Ihrer Kunden dem Link folgt. Mehr Effekt hat es, die Präsentation gleich in die Seite einzubinden. Das ist mit Slideshare leicht möglich: Hierzu gibt es einen „Embed-Code“ (1), einen Code, den Sie in die Zwischenablage kopieren (2) und dann in den Quelltext des WeblogEintrages oder der Homepage einbinden können.

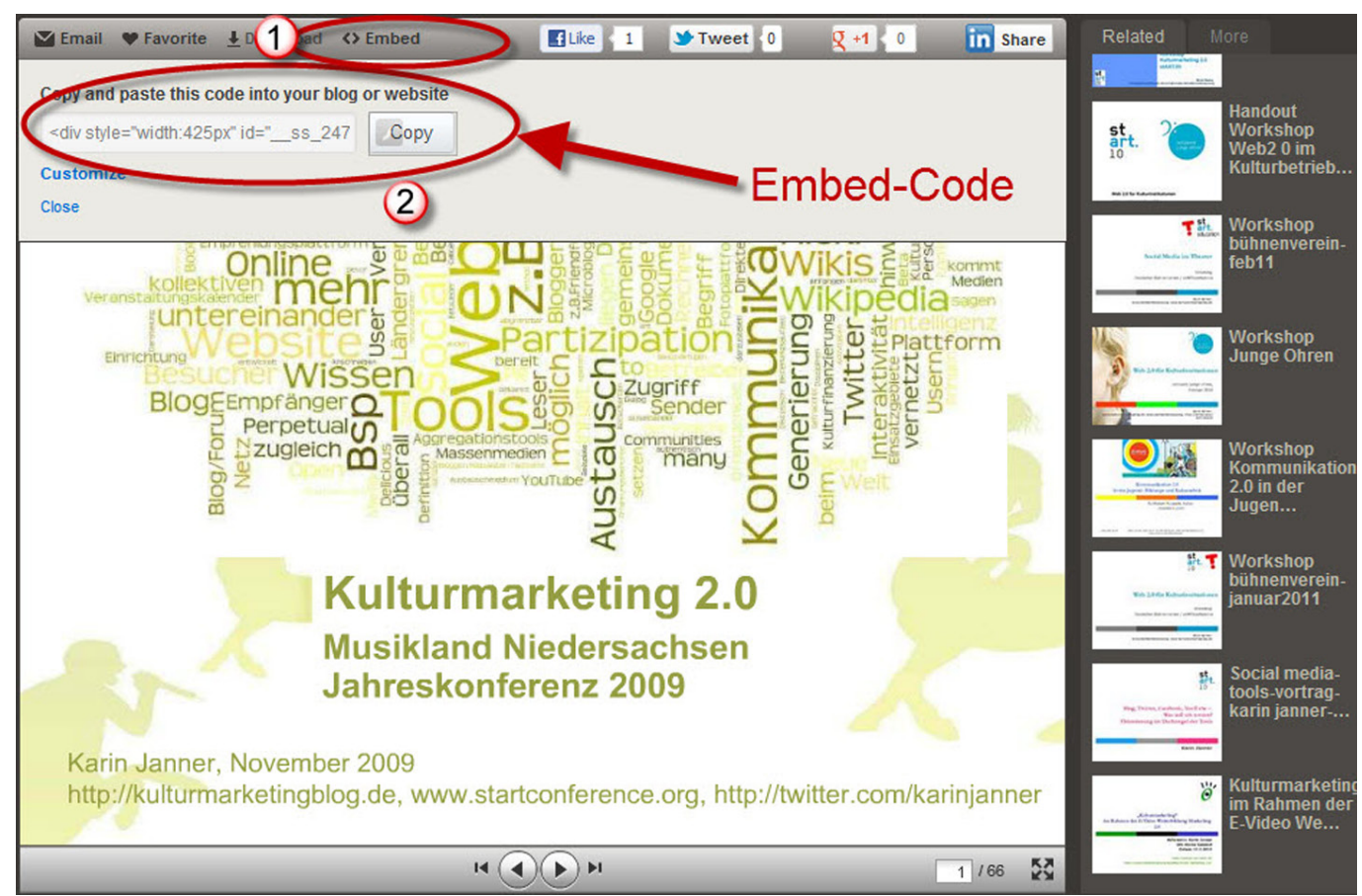

Bei der Homepage kommt es darauf an, ob Ihre IT das zulässt. Technisch möglich ist es allemal!

\subsubsection{Slideshare als Repositorium}

Sie eröffnen sich hier einen Account und haben dann die Möglichkeit, Präsentationen nach Slideshare hochzuladen. Was bringt Ihnen das?

- Ihre Präsentationen sind nicht nur zu einem bestimmten Termin (bei einer Veranstaltung oder einer Arbeitsgruppensitzung) zugänglich, sondern frei im Netz. Damit können Sie die Zahl derer, die die Präsentation zur Kenntnis nehmen, vervielfachen, insbesondere, wenn Sie Werbung für diese Präsentation machen. Sie haben damit die Reichweite örtlich und zeitlich vergrößert und erreichen gegebenenfalls ganz andere Zielgruppen.

- Sie bieten damit „Open Educational Resources“ (OERs) an, freie Lern- und Bildungsinhalte.

- Sie haben eine automatische Sicherung Ihrer Präsentationen.

- Sie können die Präsentationen mit Hilfe eines Embed-Codes ohne Weiteres auf Ihrer Webseite, in einem Weblog oder Wiki einbauen, so dass die Präsentation dort angesehen werden kann.

- $\quad$ andere erfahren über RSS-Feeds oder Recherche von Ihrer Präsentation und haben die Option, diese in ihre eigenen Webpräsenzen einzubauen. 
Sie merken: Der Wirkungsgrad einer Präsentation, die bei Slideshare hochgeladen wird, ist eine ganz andere, als wenn man sie bei einer Veranstaltung einem begrenzten Publikum zeigt. Das muss nichts heißen, bleiben doch Inhalte im Netz oft unentdeckt, aber potentiell haben andere Nutzer die Möglichkeit, über Recherche, RSS-Feed oder Abonnement auf Ihre Präsentation zu stoßen, sie zur Kenntnis zu nehmen und gegebenenfalls bei sich in das Web- oder Kursangebot mit einzubauen. Plagiat? Nein. System: Gute Inhalte finden weitere Verbreitung als sonst möglich.

\subsection{Scribd}

Die Textablage 2.0 namens Scribd ist ganz parallel zu Slideshare zu sehen: Man legt hier Texte aller Art ab und erschließt sie mit Hilfe von Tags. Man hat so die Möglichkeit, die Inhalte zu verbreiten oder auch einzubinden auf einer Webseite oder einem Weblog.

\subsubsection{Scribd als Ressource}

Sie können auf Scribd [de.scribd.com] mit Hilfe bestimmter Stichwörter recherchieren (1), bekommen dann Ergebnisse (2) und können gegebenenfalls sehr spezifisch die Ergebnismenge eingrenzen (3).

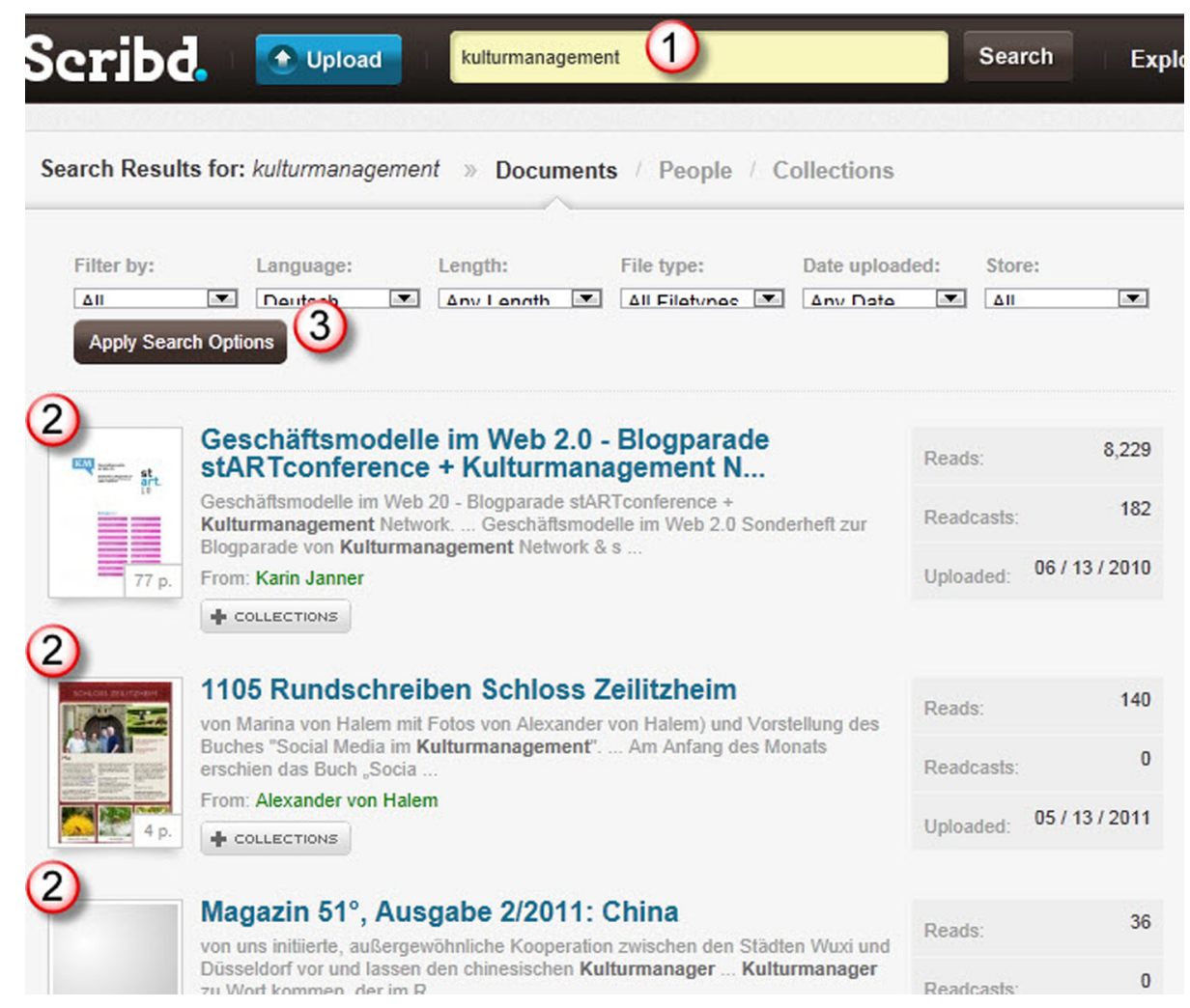

Jetzt können Sie ein Dokument öffnen und haben unten am Fuss die Möglichkeit, den Einbettungscode zu holen, mit dessen Hilfe Sie es in eine Webseite einbetten können. 


\subsubsection{Scribd als Repositorium}

Wie mit fremden Texten, kann man auch mit eigenen Texten verfahren, die man veröffentlichen will. Zunächst auf Scribd und dann kann man mit Hilfe des Einbettungscodes den Text an unterschiedlichen Orten einbinden und anbieten. Publizieren im Netz - leicht gemacht! - Hier ein Beispiel: Eine Kollegin schrieb schon in Studienzeiten in einem eigenen Weblog, und zu Bewerbungszwecken erstellte sie einen Eintrag, der ihre Magisterarbeit beschrieb, quasi als Visitenkarte ihrer fachlichen Kompetenz. Und für jene Personalverantwortlichen, die es ganz genau wissen wollten, setzte sie in einem Fenster darunter ihre ganze Magisterarbeit ein. Die Arbeit lag auf Scribd, das Fenster war durch einen einfachen Einbettungs-Code (,embed code“) erstellt worden. Das wirft ein Schlaglicht auf die Möglichkeiten, die das Publizieren von Inhalten mit Hilfe von Web 2.0-Software gewonnen hat. Gegebenenfalls braucht man keinen Verleger, kein Book on Demand, keinen Volltextserver mit einer policy, die wieder Schranken aufstellt, man benötigt nur einen Web 2.0-Dienst, mit dessen Hilfe man sofort publizieren kann. In diesem Fall war es Scribd, das ist sinnvoll, weil hier viele nach Texten suchen, es hätte aber auch ein Wiki oder Weblog sein können, wo man auf einem Eintrag oder einer Seite einen beschreibenden Text hätte platzieren und die Datei hochladen und verlinken können. Wenn man um die Möglichkeiten weiß, ist alles kein Aufwand. - Übertragen Sie es auf Ihre Verhältnisse: Wenn Sie Texte zu veröffentlichen haben, ist Scribd eine gute Möglichkeit dafür. Wenn es aktueller oder vielfältiger sein soll, sind Weblogs oder Wikis in Betracht zu ziehen.

\subsection{Flickr}

Flickr [www.flickr.com] ist einer von vielen sozialen Foto-Repositorien, vielleicht der älteste, vielleicht der umfangreichste. Mittlerweile gehört er schon lange zu Yahoo! und bietet die Möglichkeit,

1. diesen Fotodienst einfach als Betrachter und Verwender zu nutzen, indem man dort nach Stichworten recherchiert und Fotos nach Schlagwörtern, Stichwörtern aus Titel oder Beschreibungen und/oder Gruppen(-themen) findet.

2. diese Plattform als Möglichkeit der Ablage und/oder des Publizierens nutzt, dort Fotos hochlädt, erschließt und sie unterschiedlichen Kreisen oder öffentlich zugänglich macht und gegebenenfalls sie von dort aus weiterverwertet.

$\mathrm{Zu}$ beiden Punkten im Folgenden etwas eingehender.

\subsubsection{Flickr als Ressource}

Interessanterweise muss man im Bereich sozialer Medien nicht unbedingt selbst produzieren, um sie nutzen zu können. Auf Flickr laden nicht nur Privatpersonen ihre Fotos hoch, die einer Weiternutzung ihrer Fotos zustimmen, es sind auch viele Institutionen, die Flickr als Repositorium verwenden. Da zu den einzelnen Bildern jeweils die Lizenz angegeben ist, kann man nach „freien“ Bildern suchen, die man weiter verwenden kann. 
CC-Lizenzen

1. Verschaffen Sie sich einen Überblick mit Hilfe der Seite von Creative Commons über die verfügbaren Lizenzen: [creativecommons.org/licenses/?lang=de]

2. Wenn Sie eine Lizenz gefunden haben, die zu passen scheint, klicken Sie „Die Lizenz-Deed ansehen" an und überprüfen Ihren ersten Eindruck noch einmal anhand der (englisch) ausformulierten Lizenz

3. Wenn diese wirklich passt, klicken Sie unten auf „Use this licence for your own work“. Sie kommen nun auf eine Seite, wo Sie per Knopf das Aussehen der Lizenz, wie Sie sie dann in Ihre Homepage einbinden, auswählen können

4. Wenn Sie gewählt haben, markieren Sie den angegrauten Text in der Box unter „Have your own website?“, kopieren ihn mit Strg/Ctrl+c in die Zwischenablage und fügen ihn in Ihrer Homepage/Ihrem Weblog/Ihrem Wiki an der gewünschten Stelle ein. Sie müssen sich dafür in der Quellcode-Ansicht befinden, damit es richtig angezeigt wird, denn wenn Sie das in der WYSIWYG-Ansicht (What you see is what you get - also in einem Editor) einfügen, bekommen Sie in der Darstellung nur Zeichensalat zu sehen.

Wenn Sie eine Veranstaltungsreihe zu einem bestimmten Thema haben, ist Flickr neben freien, hybriden und kommerziellen Bilderdiensten durchaus eine Alternative, um an passendes Bildmaterial zu bekommen, das man weiter verwerten kann.

Recherchieren Sie hierzu einfach mit Stichworten aus Ihrem Thema. Wenn Sie beispielsweise irgendwann eine Ausstellung zum Thema Quilts organisieren, in einem Newsletter schon einmal einen Hinweis bringen möchten und noch kein Foto zur Hand haben, dann können Sie in Flickr nach „quilt“ oder „quilts“ suchen. Bei den Ergebnissen schauen Sie nur insoweit hin, ob es passt oder nicht, dann klicken Sie auf „erweiterte Suche“.

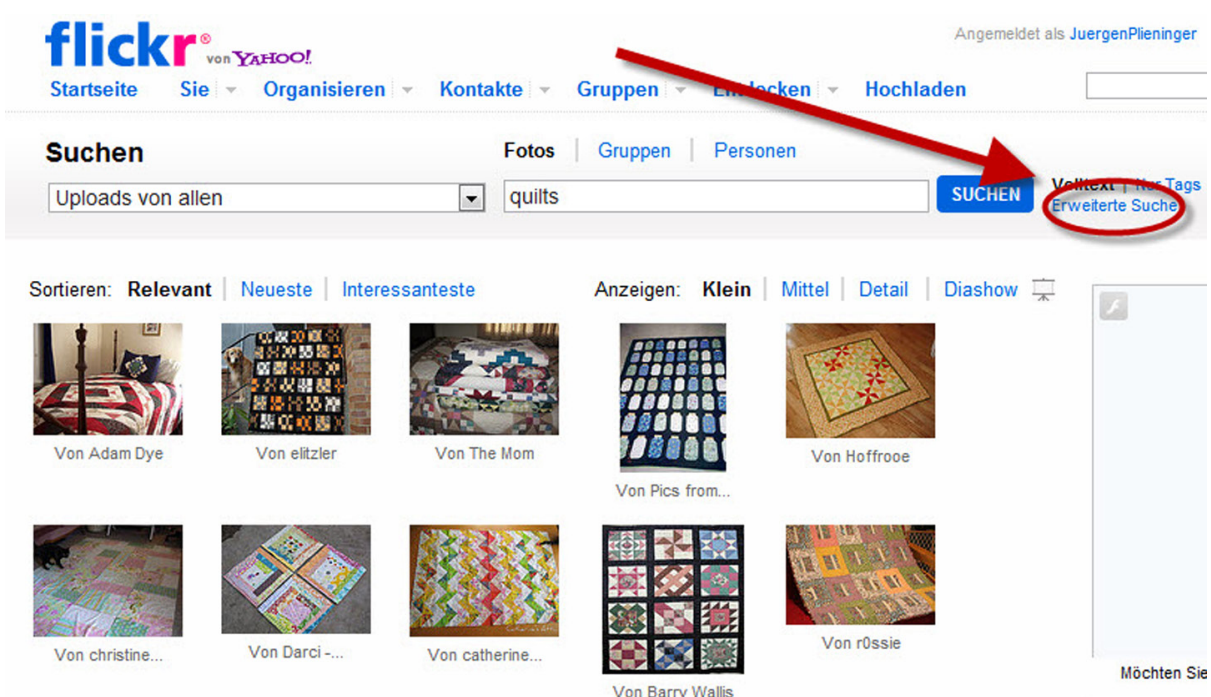


Hier haben Sie ganz unten die Möglichkeit, nach bestimmten CC-Lizenzen zu suchen. Wenn Sie jetzt die Häkchen bei CC-Lizenz, bei kommerziell und bei Wiederverwendung setzen,

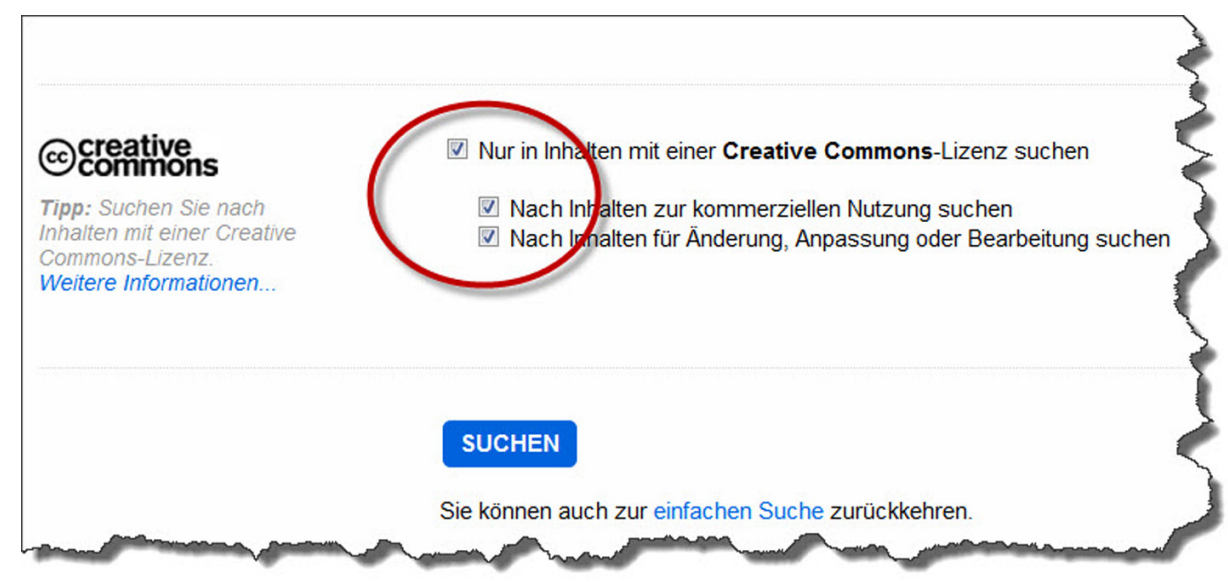

bekommen Sie Ergebnisse angezeigt, die mit einer Lizenz ausgestattet sind, die eine Weiterverwendung erlauben, wenn Sie den Namen der Flickr-Nutzerin/des FlickrNutzers angeben und die Lizenz dazusetzen. Wie das geht, haben wir oben in der Checkliste zu den CC-Lizenzen beschrieben.

\subsubsection{Flickr als Repositorium}

Sie können Flickr auch als Ablage- und Weiterverwendung benutzen. Legen Sie sich dazu einen Account an, das geht auch mit einem Google- oder Facebook-Account.

\section{flickr}

\section{Fast fertig!}

Wählen Sie Ihren neuen Flickr Benutzernamen.

Sie können diesen jederzeit ändern. Leerstellen sind zulässig.

\section{MEINEN ACCOUNT ERSTELLEN}

Die Community ist der wichtigste Bestandteil von Flickr. Stellen Sie sicher, dass Sie unsere Community-Richtlinien kennen 
Sie können sich ein Profil anlegen, Kontakte auswählen und Gruppen gründen/beitreten:

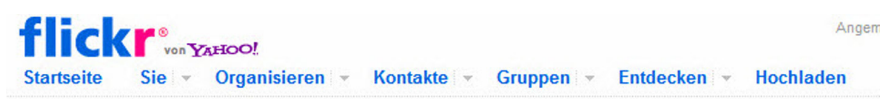

\section{- Hej JuergenPlieninger!}

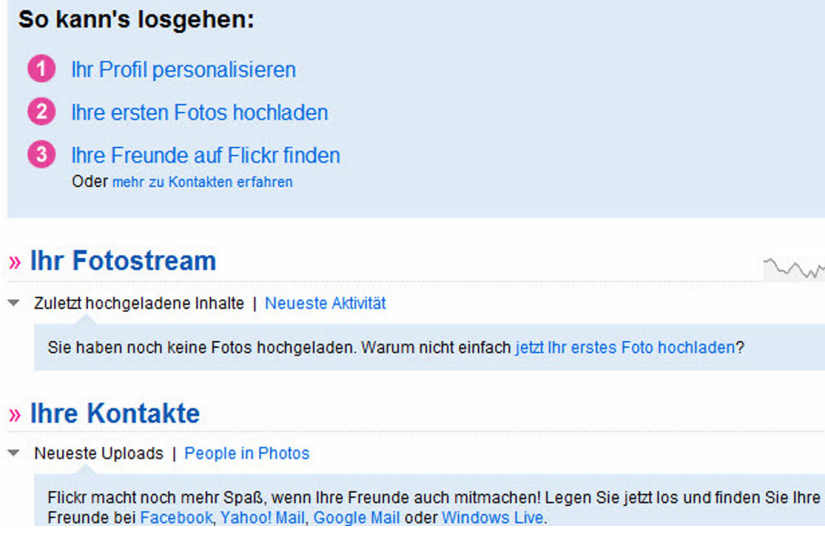

und können Fotos hochladen und erschließen.

flickrron

Startseite Sie * Organisieren - Kontakte - Gruppen - Entdecken * Hochladen

\section{Upload auf Flickr}

Sie haben $\mathbf{0} \%$ Ihres Foto-Limits von 300,00 MB und 0 Ihres Video-Limits von 2 für diesen Monat erreicht. Möchten Sie ein Upgrade durchführen?

\section{Fotos und Videos auswählen мะu}

Hinweis: Videos sind auf eine Länge von 90 Sekunden und auf eine Dateigröße von 150MB beschränkt. Weitere Informationen...

Schritt 2:

Hochladen

Schritt 3:

Titel, Beschreibungen oder Tags bzw. zu einem Album hinzufügen

Upload-Tools

Wir haben Tools für Windows und Mac entwickelt, mit denen Sie Ihre Fotos und Videos schnell und effizient von Ihrem Desktop aus auf Flickr hochladen können.

Auf der Flickr Tools-Seite finden Sie weitere Informationen und Downloads.

PSST! Auf der Suche nach unserem Basis-Uploader? 
Die Fotos lassen sich gut mittels Beschreibung und Schlagwörtern erschließen.

\section{Diesen Upload beschreiben}

Alternativ können Sie sie in Organizr öffnen, um eine feiner abgestufte Kontrolle zu haben.

Serienbearbeitung

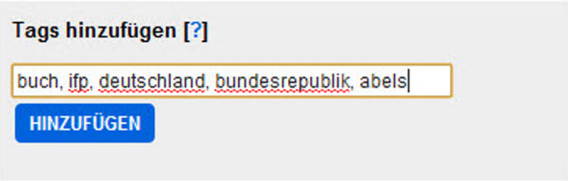

Titel, Beschreibungen und Tags

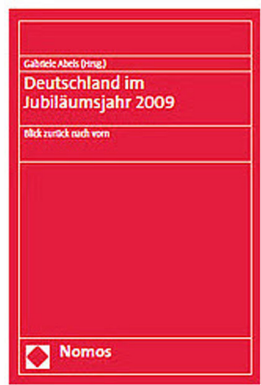

Zu Album hinzufügen

Sie haben noch keine All

Sie können weiterhin sehr fein einstellen, mit welchen Rechten das Foto ausgestattet wird, was die Sichtbarkeit gut abstufbar macht.

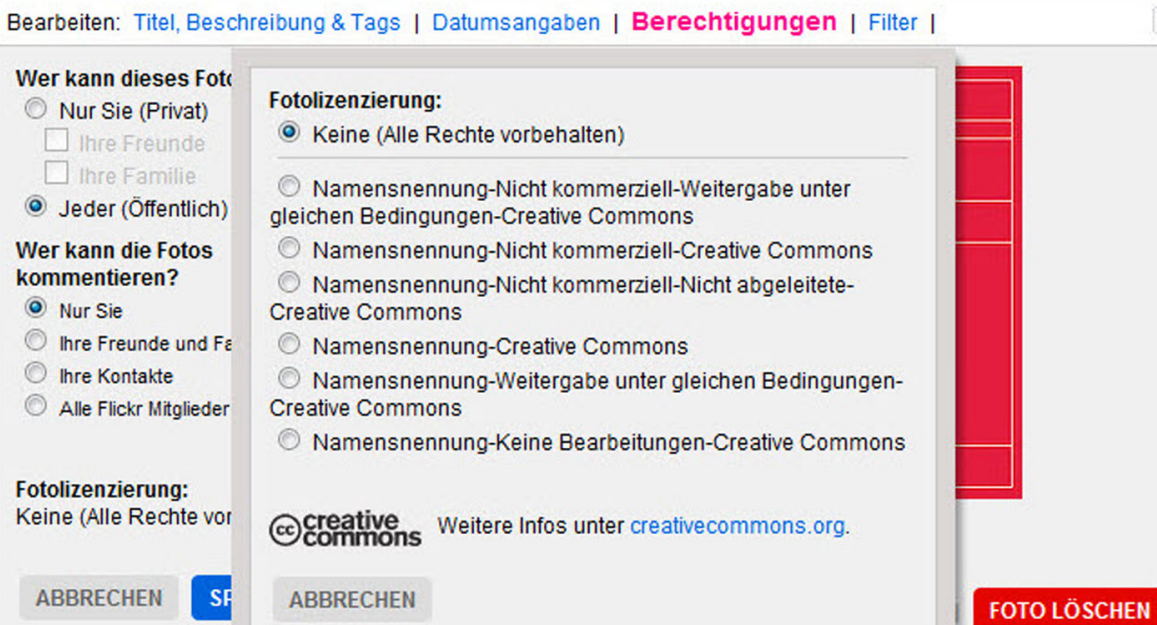

(1) Namensnennung-Nicht kommerziell-Weitergabe unter gleichen Bedingungen-Creative Commons

Namensnennung-Nicht kommerziell-Creative Commons

Namensnennung-Nicht kommerziell-Nicht abgeleiteteCreative Commons

Namensnennung-Creative Commons

Namensnennung-Weitergabe unter gleichen BedingungenCreative Commons

Namensnennung-Keine Bearbeitungen-Creative Commons

creative Weitere Infos unter creativecommons.org.

Sie können nun die eigenen Fotos, die jeweils eine spezifische Webadresse haben, für die eigenen Webpublikationen - sprich: Homepage, Twitter, Weblog, Facebook etc. verwenden, indem Sie - anstatt das Bild auf jeden dieser Dienste hochzuladen - es nun per Webadresse (URL) einbinden. Das spart Arbeitszeit.

Sie können Ihre Bilder in Alben zusammen fassen. So kann man beispielsweise ein Album „Veranstaltungen“ anlegen und von jeder Veranstaltung der Institution einige Bilder hinzufügen, so dass ein Gesamtbild von den Veranstaltungen der letzten Zeit entsteht. 
Man kann auch die „soziale Funktion“ von Flickr ausnützen, indem man für bestimmte Zwecke eine Gruppe einrichtet und von verschiedenen Accounts aus diese Gruppe mit Bildern, sprich: mit Leben füllt.

\subsection{Youtube}

Youtube [www.youtube.com] kann man unter denselben Vorzeichen verwenden wie Flickr, nur dass es sich hier um Videos und nicht um Bilder handelt. Und dass es schwieriger ist, die entsprechende Lizenz herauszubekommen. Das zu Google gehörende Youtube ist einer von vielen Videodiensten.

Betrachten wir wiederum zwei Seiten, diejenigen des Nutzers und die des Produzenten.

\subsubsection{Youtube als Ressource}

Wenn Sie Youtube-Videos nutzen möchten, beispielsweise durch Einbettung in einen Beitrag eines Weblogs oder auf der Homepage Ihrer Institution, gibt es keine so differenzierte Suchmöglichkeit wie bei flickr. Sie können also - um beim obigen Beispiel zu bleiben - nach „quilt“ oder „quilts“ suchen und bekommen dann Ergebnisse, die in der Beschreibung sowohl „Standard Youtube-Lizenz“ als auch „lizensiert nach Creative Commons“ stehen haben. Hier ein Beispiel mit der Standardlizenz:

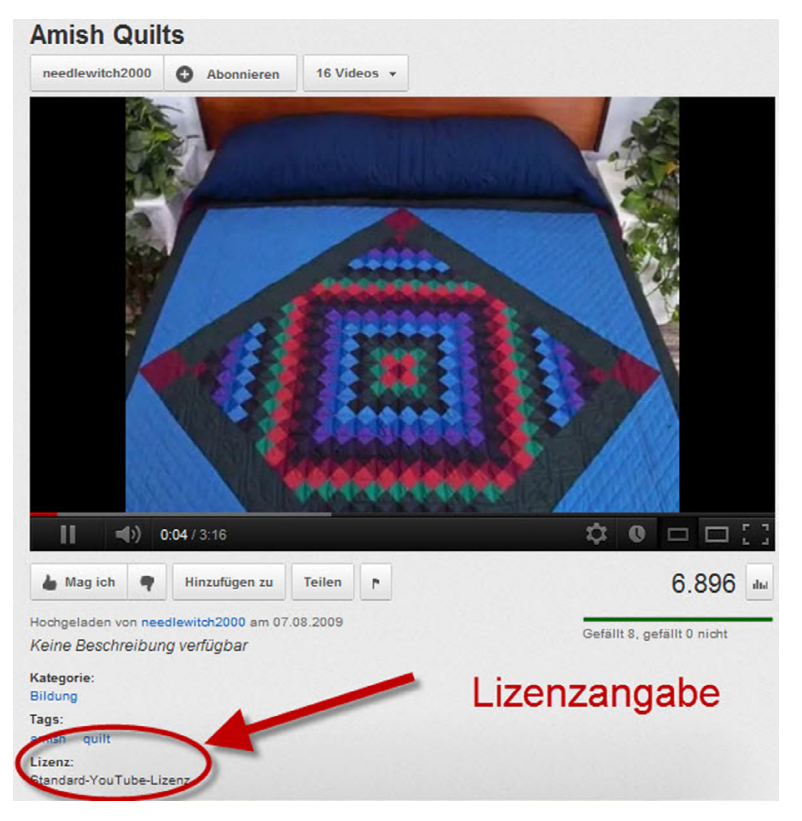

Und es ist sehr zeitaufwändig, die Videos mit CC-Lizenzen herauszufinden, da keine erweiterte Suche angeboten wird. Was tun? Wenn Sie die Spezialsuchmaschine CC-Search [search.creativecommons.org/] einsetzen, können Sie zwar die Suche auf Youtube einschränken, sehen aber bei den Ergebnissen, dass die Suche nicht auf die Lizenzform einschränkt, sondern lediglich eine Suche innerhalb der Beschreibung der Videos ist. So bekommen Sie auch Filme in der Ergebnisliste angezeigt, die in der Beschreibung „Musik: Creative Commons“ stehen haben, in ihrem Fall also bezieht sich das „CC“ auf die Begleitmusik.

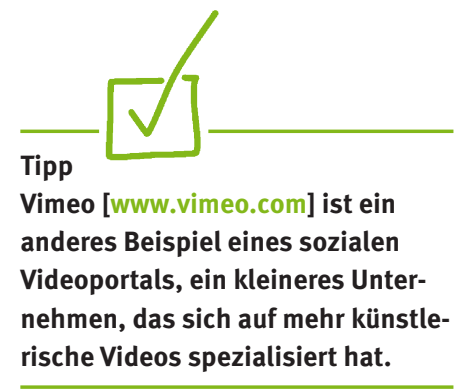


Wenn Sie daher einen Film in Ihre Homepage, Ihr Blog oder in Ihre institutionelle Facebook-Seite einbinden wollen, müssen Sie das entweder per Verlinkung tun (gänzlich risikofrei) oder per Einbettung des Videos in Ihren Rahmen. Das Video wird dann praktisch von Youtube bezogen und von dieser Plattform abgespielt. Das ist risikoarm, da die Plattform ja extra einen Embed-Code herstellt, damit diese Inhalte auch anderswo eingebunden werden können.

Abmahnungen in diesem Feld sind (noch) nicht bekannt, als erstes wäre die Video-Plattform im Rahmen der so genannten „Störerhaftung“ betroffen. Sie können also Videos von Youtube und auch Vimeo einigermaßen risikolos beziehen, zumal wenn Sie die Quelle benennen.

Technisch geht das so:

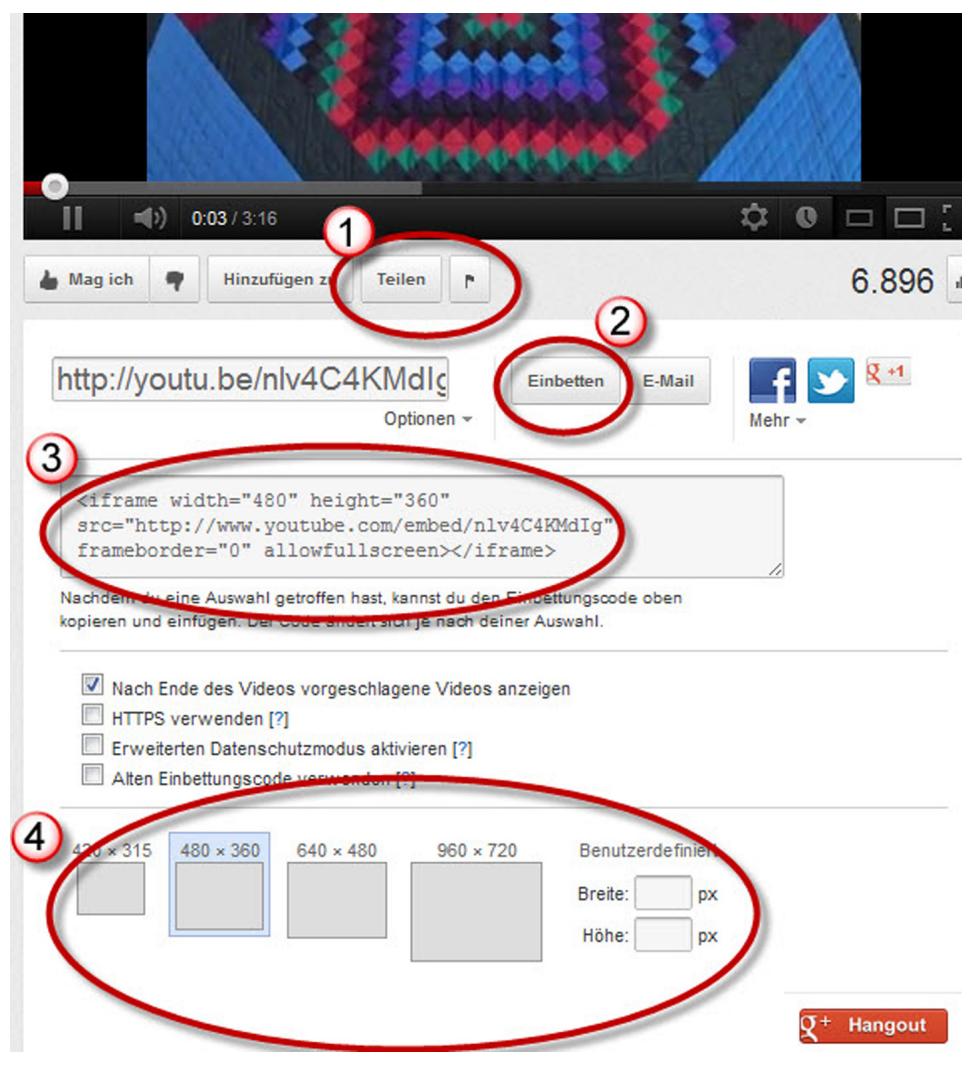

Unter dem Video finden Sie den Knopf „Teilen“ (1), dort wird dann der Knopf „Einbetten“ (2) angezeigt, worauf in einem Kasten unterhalb der Code in angegrauter Schrift angezeigt wird (3) und mit copy \& paste in die Zwischenablage übernommen werden kann. Unten (4) finden Sie noch etliche Optionen, mit denen der angezeigte Code verändert wird, beispielsweise, wenn Sie die Größe des eingebetteten Videos beeinflussen wollen. 


\subsubsection{Youtube als Repositorium}

Wenn Sie oder Ihre Institution Videos auf Youtube oder Vimeo hochladen wollen, müssen Sie sich wieder einen Account anlegen bzw. Sie können einen vorhanden Google-Account nutzen. Sie bekommen dann nach dem Einloggen die Option „Video hochladen“ ganz oben angezeigt,

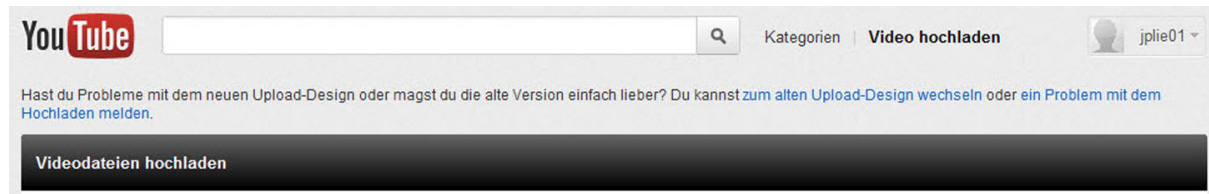

Dateien von deinem Computer auswählen

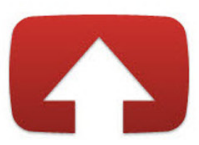

Verschiebe Videos per Drag \& Drop an eine beliebige Stelle auf dieser Seite, um mit dem Hochladen zu beginner.

Lade HD-Videos in verschiedenen Formaten und mit bis zu 15 Minuten Lange hoch. Erhohe dein Limit

und haben dann die Möglichkeit, die hochgeladenen Filme mit der CC-Lizenz auszustatten, so dass diese bei der Anzeige des Films mit angezeigt wird. Ebenso haben Sie die Möglichkeit, Filme von anderen Youtube-Nutzern mit CC-Lizenz zu verwenden, zu adaptieren und in andere Filme einzubinden. Wo jeweils diese Lizenzen angezeigt und ausgewählt werden können, zeigt ein Film „Neuer Youtube-Button - Wiederverwendung erwünscht!“ von Y2Bus [http://www.youtube.com/watch?v=BBudIKqS6RM].

Hier können Sie also als Produzent agieren und Filme bearbeiten. Sie hätten damit die Möglichkeit, Videos in ihrem „Kanal“ aufzulisten und gegebenenfalls auch Sequenzen anderer Videos in Ihre zu übernehmen.

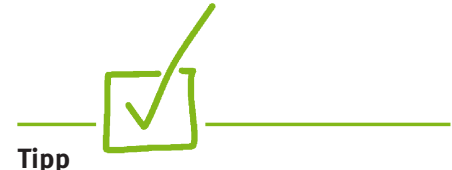

Informationen zu Youtube und Creative Commons-Lizenzen bekommen Sie auch textorientiert von Youtube selbst [www.youtube. com/t/creative_commons] 